\title{
Astrometric exoplanet surveys in practice
}

\author{
Johannes Sahlmann ${ }^{1,2}$ \\ ${ }^{1}$ European Space Agency, ESAC, P.O. Box 78, Villanueva de la Cañada, 28691 Madrid, Spain \\ ${ }^{2}$ European Space Agency, STScI, 3700 San Martin Drive, Baltimore, MD 21218, USA \\ email: Johannes.Sahlmann@esa.int
}

\begin{abstract}
Conversely to the transit photometry and radial velocity methods, the astrometric discovery of exoplanets is still limited by the sensitivity of available instruments. Ground-based surveys are now sensitive to giant planets in orbit around nearby low-mass stars and brown dwarfs. In 2014, ESA's Gaia mission began its survey, which is expected to discover thousands of giant exoplanets by detecting the astrometric orbital motions of the host stars.
\end{abstract}

Keywords. Astrometry, planetary systems, binaries, telescopes, space vehicles

\section{Introduction}

In comparison to other observational techniques, the contributions of astrometry to the discovery of exoplanets have so far been limited (Fischer et al. 2014). However, promising results were obtained with past (Muterspaugh et al. 2010) and ongoing surveys.

\section{Ongoing surveys}

Most present-day observational programs employ optical/infrared cameras on intermediate and large telescopes (e.g. Boss et al. 2009; Lurie et al. 2014; Sahlmann et al. 2014). Typical targets are low-mass stars and brown dwarf within tens of parsec of the Sun and the planet detection sensitivities reach Jupiter-mass for year-long periods (e.g. Weinberger et al. 2014; Sahlmann \& Lazorenko 2015).

\section{Gaia}

The combination of our current knowledge of giant planet occurrence with the astrometric precision, sampling, and 5-year duration of the all-sky Gaia mission (de Bruijne 2012) translates into a number of expected exoplanet discoveries in excess of several thousand (Casertano et al. 2008; Sozzetti et al. 2014; Perryman et al. 2014; Sahlmann et al. 2015). This major step in instrumental capabilities will have to be matched by improved algorithms that optimally exploit the data. Using very precise ground-based astrometry we employed genetic and MCMC algorithms and proved them to be efficient in constraining all astrometric parameters of a low-mass binary system (Sahlmann et al. 2013). Similar algorithms will be applied to some of the Gaia exoplanet data (cf. Sozzetti 2013), which makes them important tools for harvesting the results of the first major astrometric exoplanet survey.

\section{References}

Boss, A. P., Weinberger, A. J., Anglada-Escudé, G., et al. 2009, PASP, 121, 1218

Casertano, S., Lattanzi, M. G., Sozzetti, A., et al. 2008, A\&A, 482, 699 
de Bruijne, J. H. J. 2012, ApESSS, 341, 31

Fischer, D. A., Howard, A. W., Laughlin, G. P., et al. 2014, Protostars and Planets VI Lurie, J. C., Henry, T. J., Jao, W.-C., et al. 2014, AJ, 148, 91

Muterspaugh, M. W., Lane, B. F., Kulkarni, S. R., et al. 2010, AJ, 140, 1657

Perryman, M., Hartman, J., Bakos, G. Á., \& Lindegren, L. 2014, ApJ, 797, 14

Sahlmann, J. \& Lazorenko, P. F. 2015, MNRAS, 453, L103

Sahlmann, J., Lazorenko, P. F., Ségransan, D., et al. 2013, A\&̛A, 556, A133

Sahlmann, J., Lazorenko, P. F., Ségransan, D., et al. 2014, A\&\&A, 565, A20

Sahlmann, J., Triaud, A. H. M. J., \& Martin, D. V. 2015, MNRAS, 447, 287

Sozzetti, A. 2013, European Physical Journal Web of Conferences, 47, 15005

Sozzetti, A., Giacobbe, P., Lattanzi, M. G., et al. 2014, MNRAS, 437, 497

Weinberger, A. J., Boss, A. P., \& Anglada-Escudé, G. 2014, IAU Symposium, 299, 230-231 